\title{
MicroRNAs and Alzheimer's Disease Mouse Models: Current Insights and Future Research Avenues
}

\author{
Charlotte Delay ${ }^{1,2}$ and Sébastien S. Hébert ${ }^{1,2}$ \\ ${ }^{1}$ Axe Neurosciences, Centre de Recherche du CHUQ (CHUL), Québec, QC, Canada G1V4G2 \\ ${ }^{2}$ Département de Psychiatrie et de Neurosciences, Faculté de Médecine, Université Laval, Québec, QC, Canada G1V0A6
}

Correspondence should be addressed to Sébastien S. Hébert, sebastien.hebert@crchul.ulaval.ca

Received 25 May 2011; Accepted 13 June 2011

Academic Editor: Marcella Reale

Copyright (c) 2011 C. Delay and S. S. Hébert. This is an open access article distributed under the Creative Commons Attribution License, which permits unrestricted use, distribution, and reproduction in any medium, provided the original work is properly cited.

\begin{abstract}
Evidence from clinical trials as well as from studies performed in animal models suggest that both amyloid and tau pathologies function in concert with other factors to cause the severe neurodegeneration and dementia in Alzheimer's disease (AD) patients. Accumulating data in the literature suggest that microRNAs (miRNAs) could be such factors. These conserved, small nonproteincoding RNAs are essential for neuronal function and survival and have been implicated in the regulation of key genes involved in genetic and sporadic AD. The study of miRNA changes in AD mouse models provides an appealing approach to address the causeconsequence relationship between miRNA dysfunction and AD pathology in humans. Mouse models also provide attractive tools to validate miRNA targets in vivo and provide unique platforms to study the role of specific miRNA-dependent gene pathways in disease. Finally, mouse models may be exploited for miRNA diagnostics in the fight against AD.
\end{abstract}

\section{Introduction}

Alzheimer's disease (AD) is the most common form of dementia worldwide. It is characterized by the accumulation of extracellular amyloid (senile) plaques, composed mainly of $\mathrm{A} \beta$ peptides, and intracellular neurofibrillary tangles, containing abnormally aggregated and hyperphosphorylated Tau protein. The $\mathrm{A} \beta$ peptides are generated by the sequential cleavage of Amyloid precursor protein (APP) by $\beta$-secretase (BACE1-dependent) and $\gamma$-secretase (PSEN/presenilin-dependent). Based on the discovery of rare familial forms of AD caused mutations in the APP and PSEN genes, Hardy and Selkoe proposed the "amyloid cascade hypothesis", which suggests that $A \beta$ overproduction alone is sufficient to trigger the molecular events leading to both tangle formation and neuronal cell death $[1,2]$. Over the past two decades, several breakthroughs have been made with regard to modeling $\mathrm{AD}$ pathology in vivo in mice, providing important tools for various areas of basic and translational research. Several AD mouse models harboring human APP, PSEN, and/or MAPT (Tau) transgenes support to some extent the amyloid cascade hypothesis; however, recent clinical trials in humans suggest that amyloid-dependent signaling pathways are insufficient to cause the severe neurodegeneration and dementia in $\mathrm{AD}$ patients [3-5]. It is therefore perhaps not surprising that most, if not all, $\mathrm{AD}$ mouse models, albeit displaying massive $\mathrm{A} \beta$ deposits and/or tangles, do not recapitulate the full-blown neuropathological biochemical, cellular, and morphological changes observed in $\mathrm{AD}$ brain [6-8]. This opens the door to the identification of novel factors important for $\mathrm{AD}$ development, which could equally serve as potential diagnostic and therapeutic tools.

The past years have witnessed an explosion of papers linking microRNA (miRNA) dysfunction to human disease, including cancer, cardiology, liver disease, viral infection, and many others. Perhaps expectedly, mounting evidence also involve miRNAs in neurodegenerative disorders, with potential implications in $\mathrm{AD}[9,10]$, Parkinson's disease (PD) [11], Huntington's disease (HD) [12, 13], frontotemporal dementia (FTD) [14, 15], and amyotrophic lateral sclerosis (ALS) [16]. Because of the rapid growth of miRNA research in general, it is likely other neurodegenerative disorders will soon be added to this list. 
Recent RNA deep sequencing efforts have identified more than 1400 miRNA genes in the human genome (700 in mice) (miRBase.org). Several of these are specifically expressed in the brain, where they are proposed to function in neuronal processes such as neurite outgrowth and synapse formation [17, 18]. The biogenesis and mode of action of miRNA molecules in mammals is complex and has extensively been reviewed elsewhere (see, e.g., [19-22]). In short, miRNA precursors (pre-miRs) are cleaved in the cytoplasm by the RNase Dicer to produce small ( $21 \mathrm{nt}$ in length) single-stranded nonprotein-coding RNAs. These latter function as part of the RNA-induced silencing complex (RISC), which targets specific mRNA transcripts with imperfect complementarity. Binding of the miRNA to its target leads to translation inhibition and/or mRNA degradation $[23,24]$, which ultimately leads to downregulation of the encoded protein. It is predicted that 25 to $70 \%$ of all protein-coding genes can be regulated by miRNAs, depending on the developmental, cellular, and/or physiological context. Moreover, each miRNA can target up to several hundred transcripts in vivo, thus potentially regulating multiple biological pathways, including those implicated in neuronal survival [25]. It is therefore not surprising that genetic ablation of Dicer in the brain, which leads to an overall reduction in miRNA production, results in rapid neurodegeneration [26-28].

The somewhat humble introduction of miRNAs in the AD field came in 2007 when Lukiw studied the expression levels of 13 brain miRNAs in control and AD patients, some of which were specifically altered in disease [29]. Since then, several groups have performed global miRNA expression analysis of AD brain $[9,10,29-31]$ and peripheral system $[32,33]$. These profiling experiments tend to show a trend towards the identification of "AD or neurodegenerative disease-specific" miRNAs, including miR-29, miR-9, miR15a, miR-181c, miR-101, miR-106b, miR-146a, and miR107 , which have been identified in two or more independent studies. Interestingly, several of these miRNAs may have a direct role in modulating APP (miR-106, miR101) [34-36] or BACE1 (miR-29, miR-107) [9, 37-39] expression, therefore potentially contributing to increase amyloid production. Because (1) miRNAs regulate APP and BACE1 expression, whose increased protein levels are linked to genetic and sporadic $\mathrm{AD}$, respectively (reviewed in $[21,40])$ and (2) miRNAs control several pathways involved in neuronal function, inflammation and survival, miRNA research provides an interesting new perspective to study the underlying mechanisms involved in $\mathrm{AD}$ development.

\section{2. miRNA-Deficiency and the AD Brain}

While depleting all miRNAs remain a conceptually crude approach, it is interesting to observe several parallels between Dicer-deficient brain and AD brain. For instance, neuronal Dicer conditional knockout (cKO) mice develop progressive neurodegeneration, have reduced brain size, enlarged ventricles, neuroinflammation, apoptosis (in some cases), as well as impaired dendritic branching, and spine length
$[26,41,42]$. In addition, neuronal Dicer cKO mice exhibit AD-like hyperphosphorylation of endogenous tau [26], which is not observed in nontransgenic mice. These latter results succeed previous studies in the fly linking tau toxicity to miRNA dysfunction [43]. Interestingly, specific loss of Dicer in oligodendrocytes results in axonal degeneration accompanied by abnormal axonal transport and endogenous APP accumulation [44]. This model also displays signs of oxidative stress, and, taken together, these results point out the importance of Dicer and miRNAs in maintaining neuronal function. Although controversial, one study suggests that neuronal Dicer deficiency promotes learning and memory, at least at stages prior to neuronal loss [45]. How these observations translate to human disease remains speculative, but nevertheless provides "proof-of-principle" that loss (and perhaps gain) of brain miRNA function can participate in several neuropathological features of $\mathrm{AD}$.

\section{3. miRNA Profiles from AD Mice}

Apart from the more obvious role of miRNAs in regulating the expression of disease-related genes (e.g., APP and BACE1), it is likely that a combination of more subtle (direct or indirect) mechanisms alter disease progression over years, possibly decades. As example, sustained miR29 deficiency may not only increase BACE1 and A $\beta$ levels, but also affect DNA methylation and neuronal survival [46, 47]. In addition, it remains difficult to predict whether the observed changes in miRNA levels in humans are a cause or consequence of the neurodegenerative process. The study of miRNA expression profiles in AD mouse models may help to address these questions.

Wang et al. were the first to study global miRNA profiles from $\mathrm{AD}$ mice using microarrays [48]. For this, they used the APPSwe-PS1M146L mouse model. Of the 37 differently expressed miRNAs, several (miR-20a, miR29a, miR-125b, miR-128a, and miR-106b) miRNAs were significantly downregulated, while others (miR-34a, let-7, miR-28, and miR-98) were upregulated. Interestingly, some miRNAs were similarly shown to be affected in AD brain in humans, including miR-29a and miR-106b [9, 30, 31, 40, 49]. It is noteworthy that miRNA alterations were measured at 3 months of age prior to $\mathrm{A} \beta$ plaque formation. In most cases, miRNA alterations were maintained or even accentuated during amyloid plaque formation at 6 months of age, therefore supporting the "cause" hypothesis. The increase in miR-34a in the mutant mice is proposed to function in regulating apoptosis via Bcl-2 modulation [48]. In a followup study, the group showed by sensitive miRNA quantitative RT-PCR that miR-106b is upregulated in 3-month-old AD mice but downregulated at 6 months [50]. These changes correlated to some extent with transforming growth factor, beta receptor II (T $\beta$ RII) expression, and a putative miR$106 \mathrm{~b}$ target gene [50]. While these studies highlight the importance of microarray validation, they also suggest a possible transient effect of $\mathrm{AD}$ pathology (in this case $\mathrm{A} \beta$ plaque formation) on miRNA expression and vice versa.

More recently, Schonrock et al. studied the effects of exogenous $A \beta$ on miRNA expression levels in mouse 
hippocampal neurons in culture [51]. Again, several miRNAs downregulated by $\mathrm{A} \beta$ treatment were previously found to be decreased in human AD brain, including miR-9, miR-181c, miR-30c, miR-148b, miR-20b, and let-7i. Of interest, certain miRNAs decreased concomitantly with $\mathrm{A} \beta$ pathology progression in vivo in APP23 mice expressing human APP751 with the K670N/M671L mutations. While these observations support the "consequence" hypothesis of miRNA dysregulation in $\mathrm{AD}$, it is noteworthy that some miRNA molecules became affected prior to $\mathrm{A} \beta$ plaque formation (like miR-409$3 p$ and let-7i) similar to what is seen in the Wang et al. study ([48], see above). Furthermore, the expression of certain miRNAs changed over time (from up- to downregulated or vice versa), again supporting the transient effect on miRNA expression during $\mathrm{AD}$ development.

While studying the role of actin and the actin-binding protein cofilin in $\mathrm{AD}$, Yao et al. observed decreased miR103 and miR-107 levels in 4-month old (A $\beta$ plaque bearing) Tg19959 mice that express mutant APP with KM670/671NL and V717F FAD mutations [52]. As mentioned above, both miR-103 and miR-107 were shown to be decreased in MCI and late-onset $\mathrm{AD}[10]$. The authors further showed that these miRNA paralogues could effectively regulate cofilin expression in vitro, providing a mechanism for the observed increase in rod-like structures in this mouse model.

Loss of presenilin function is proposed to underlie memory impairment and neurodegeneration in the pathogenesis of $\mathrm{AD}$ [53]. Interestingly, small-scale miRNA profiling from Psen1 KO mice with, as a result, reduced $\gamma$-secretase activity and $\mathrm{A} \beta$ production, showed that miR- 9 down-regulation coincided with neurodegeneration [54]. It is noteworthy that miR-9 was shown to be an important regulator of neurogenesis, both in zebrafish and mice $[55,56]$. Based on these observations, it is tempting to speculate that miR-9, which is downregulated in $\mathrm{AD}$ brain, participates actively in neuronal maintenance, and functions in a feedback loop with $\mathrm{A} \beta$.

Candidate miRNA approaches have equally been performed. For instance, Li et al. studied miR-146a expression in five different $\mathrm{AD}$ mouse models, including Tg-2576, TgCRND8, PSAPP, 3xTg-AD, and 5XFAD [57]. This group had shown earlier that miR-146a expression levels were increased in $\mathrm{AD}$ brain [58]. It turned out that miR-146a was significantly increased in age (4- to 12-month-old) when compared to young (1- to 2-month-old) mice, and this, independently of the model tested [57]. Notably, miR-146a has repeatedly been shown to be implicated in the regulation of the inflammatory response [59]. Moreover, neuroinflammation is thought to play a critical role in the pathogenesis of chronic neurodegenerative diseases including $\mathrm{AD}$ [60], evoking the hypothesis that miR-146a overexpression in these $\mathrm{AD}$ models could reflect a defense mechanism against the deleterious effects of neuroinflammation. Interestingly, synthetic $\mathrm{A} \beta$ was shown to induce miR-146a expression in cultured human neuronal (and glial) cells [58]. Taken together, the abovementioned observations suggest that miRNA-regulated gene pathways, such as the miR-146a pathway, could function both upstream and downstream of $\mathrm{AD}$ pathology (cause and consequence).

\section{4. miRNA-Mediated Regulation of AD Genes In Vivo}

One of the main challenges in the miRNA field is the identification of bona fide target genes. Several genome-wide methods are currently available to address this question, including microarray expression analyses following miRNA transfection/inhibition, Argonaute cross-linked immunoprecipitation (CLIP)-seq assays, and SILAC-based proteomic approaches [61-63]. So far, however, most miRNA studies involving AD-related genes have relied on artificial $3^{\prime}$ UTR reporter systems (e.g., luciferase-based assays) as well as single miRNA gain- or loss-of-function experiments in cells. While these methods remain indispensable in the validation of miRNA: mRNA targets, they rarely put both molecules in their physiological context. In addition, it is important to consider that some miRNA:mRNA targets are not conserved in lower organisms such as C. elegans and Drosophila, making extrapolations to mammalian brain sometimes difficult (e.g., see [43]).

As of now, most studies addressing the role of miRNAs in $\mathrm{AD}$ gene regulation have focused on APP. These have lead to the identification of at least six miRNAs that could regulate APP in vitro and in cells, including the miR-20a family (i.e. miR-20a, miR-17-5p, and miR-106a/b), miR-101, and miR-16 [40, 64-68]. Interestingly, Liu et al. showed that miR-16 inhibition in 8-month-old SAMP8 mice, a model for accelerated senescence, reduced endogenous APP levels by $\sim 50 \%$ [68]. Again, the effects on $A \beta$ production remain to be determined. While providing the first "proof-of-principle" of APP regulation by miRNAs in vivo, this study does not exclude the role of other miRNAs in this process in the brain. For instance, our preliminary data indicate that APP mRNA levels are increased in miR-20a/17-5p double KO mice (Hebert, S.S., unpublished observations). In addition, it remains uncertain whether other miR-16 homologues, that is, miR-15a, miR-15b, miR-195, and miR-495 are involved in APP regulation in vivo. Interestingly, miR-16 family members have also been implicated in endogenous tau phosphorylation in neurons [26].

While investigating the molecular mechanisms involved in endogenous BACE1 overexpression in 6-month-old (A $\beta$ plaque bearing) 5XFAD [69], O'Connor et al. observed no significant changes in miR-29a, miR-29b, and miR-9 levels, previously shown to regulate BACE1 in vitro [9], when compared to nontransgenic controls. Another study suggests however, that miR-29c (the third miR-29 family member) overexpression is sufficient to decrease endogenous BACE1 protein levels in wild-type mice [50]. The functional effects on BACE1 activity and $\mathrm{A} \beta$ production were unfortunately not evaluated in this latter model. Boissonneault et al. showed that BACE1 protein increases in 19-month-old (A $\beta$ plaque bearing) versus 4-month-old APPSwe-PS1A246E mice [70], an effect not observed in wild-type mice. Interestingly, two miRNAs shown to regulate murine BACE1 expression in vitro, that is, miR-298 and miR-328 were downregulated in this mouse model. Whether other miRNAs are affected in the 5XFAD and APPSwe-PS1A246E mice remains to be determined. Lastly, Faghihi et al. showed that 
BACE1 protein and BACE1 antisense (noncoding) transcript levels were increased in 6-week-old Tg19959 mice [71]. Whether decreased miR-103/107 levels observed in this mouse model (see Yao et al. study above) could contribute to increase BACE1 remains to be determined. Thus, among at least five miRNAs shown to target BACE1 in vitro, that is, miR-29, miR-9, miR-107, miR-298, and miR-328, no clear evidence can be drawn from these mouse models with regard to the physiological or pathological regulation of BACE1 by miRNAs in vivo.

While the abovementioned results are interesting, the overall contribution of miRNAs in BACE1 and APP expression regulation remains unclear. The use of gene knockout mice will be indispensible to make definitive conclusions with regard to the role of miRNAs or miRNA gene families in BACE1, APP, and other AD-related gene expression regulation. More transient approaches, for instance using miRNA mimics or inhibitors, will be necessary to address the causeconsequence relationship between miRNA dysregulation and $\mathrm{AD}$ progression in the brain. Thus, both classical genetics and miRNA functional tools will be necessary to fully investigate the role of miRNAs in AD mouse models.

\section{5. miRNAs as Potential Diagnostic Tools in AD}

Much advancements have been made in the cancer field, for instance, with regard to miRNAs as potential diagnostic tools, some of which are currently available in the clinic. The interest in miRNAs in this field comes from the fact that they are readably detected in human body fluids, making them attractive biological markers. In addition, miRNAs are in general quite stable when compared to protein and/or other RNAs, providing reliable markers of cell or tissue state. While a few groups have explored the role of miRNAs in blood and CSF $[32,33]$, most AD-based studies have focused mainly on soluble $A \beta$ (and its derivatives) and tau $[72,73]$. In the future, it will be interesting to combine and/or compare these species with circulating miRNAs, which can be found in body fluids including exosomes [74]. It is noteworthy that groups have already shown this applicability in mouse models for the Duchenne muscular dystrophy and liver injury $[75,76]$.

\section{Conclusions and Future Perspectives}

As summarized above, most studies addressing the role of miRNAs in $\mathrm{AD}$ pathology remain correlative in nature, and little or no definitive proof with regard to miRNA target gene regulation in vivo is currently unavailable. Addressing these issues is crucial in order to advance our knowledge of the contribution of miRNAs, if any, in AD neuropathology. Because mice are genetically homogenous in nature when compared to humans, they provide unique tools to study miRNA-regulated gene pathways in $\mathrm{AD}$ development. Human profiling studies clearly indicate that miRNA expression profiles are altered in AD brain. Whether these changes are specific to $\mathrm{AD}$ or reflect an overall loss (or gain) of miRNA function in neurodegenerative disease remains to be determined using adequate comparative analyses. The current profiling data from $\mathrm{AD}$ mice suggest that miRNA changes equally occur in disease models, yet some discrepancies still exist. Given our current state of knowledge, the role of miRNAs in $\mathrm{AD}$ development, and their applicability as diagnostic and perhaps therapeutic tools into clinic, will require extensive follow-up studies in both in vitro and animal models. Specific miRNA gene knockout and/or transgenic mouse is required to address these and other fascinating questions.

\section{Abbreviations \\ BACE1: Beta-site APP cleaving enzyme \\ FAD: Familial Alzheimer's disease \\ Tg: $\quad$ Transgenic $3^{\prime}$ UTR \\ CSF: Cerebrospinal fluid \\ 3'UTR: 3' untranslated region.}

\section{Conflict Interests}

The authors declare no conflict of interests.

\section{Acknowledgments}

This work was supported by the Scottish Rite Charitable Foundation of Canada and the Alzheimer Society of Canada (including a fellowship for C. Delay).

\section{References}

[1] J. Hardy and D. J. Selkoe, "The amyloid hypothesis of Alzheimer's disease: progress and problems on the road to therapeutics," Science, vol. 297, no. 5580, pp. 353-356, 2002.

[2] J. A. Hardy and G. A. Higgins, "Alzheimer's disease: the amyloid cascade hypothesis," Science, vol. 256, no. 5054, pp. 184-185, 1992.

[3] T. E. Golde, L. S. Schneider, and E. H. Koo, "Anti-A $\beta$ therapeutics in alzheimer's disease: the need for a paradigm shift," Neuron, vol. 69, no. 2, pp. 203-213, 2011.

[4] K. Sambamurti, N. H. Greig, T. Utsuki et al., "Targets for AD treatment: conflicting messages from $\gamma$-secretase inhibitors," Journal of Neurochemistry, vol. 117, no. 3, pp. 359-374, 2011.

[5] L.-Y. Fan and M.-J. Chiu, "Pharmacological treatment for Alzheimer's disease: current approaches and future strategies," Acta Neurologica Taiwanica, vol. 19, no. 4, pp. 228-245, 2010.

[6] C. Balducci and G. Forloni, "APP transgenic mice: their use and limitations," NeuroMolecular Medicine, vol. 13, no. 2, pp. 117-137, 2011.

[7] K. J. Bryan, H. Lee, G. Perry, M. A. Smith, and G. Casadesus, "Transgenic mouse models of Alzheimer's disease: behavioral testing and considerations," in Methods of Behavior Analysis in Neuroscience, CRC Press, Boca Raton, Fla, USA, 2009.

[8] K. H. Ashe and K. R. Zahs, "Probing the biology of Alzheimer's disease in mice," Neuron, vol. 66, no. 5, pp. 631-645, 2010.

[9] S. S. Hébert, K. Horré, L. Nicolaï et al., "Loss of microRNA cluster miR-29a/b-1 in sporadic Alzheimer's disease correlates with increased BACE $1 / \beta$-secretase expression," Proceedings of the National Academy of Sciences of the United States of America, vol. 105, no. 17, pp. 6415-6420, 2008.

[10] W. X. Wang, B. W. Rajeev, A. J. Stromberg et al., "The expression of microRNA miR-107 decreases early in Alzheimer's 
disease and may accelerate disease progression through regulation of $\beta$-site amyloid precursor protein-cleaving enzyme 1," Journal of Neuroscience, vol. 28, no. 5, pp. 1213-1223, 2008.

[11] J. Kim, K. Inoue, J. Ishii et al., "A microRNA feedback circuit in midbrain dopamine neurons," Science, vol. 317, no. 5842, pp. 1220-1224, 2007.

[12] A. N. Packer, Y. Xing, S. Q. Harper, L. Jones, and B. L. Davidson, "The bifunctional microRNA miR-9/miR-9* regulates REST and CoREST and is downregulated in Huntington's disease," Journal of Neuroscience, vol. 28, no. 53, pp. 1434114346, 2008.

[13] E. Martí, L. Pantano, M. Bañez-Coronel et al., "A myriad of miRNA variants in control and Huntington's disease brain regions detected by massively parallel sequencing," Nucleic Acids Research, vol. 38, no. 20, pp. 7219-7235, 2010.

[14] R. Rademakers, J. L. Eriksen, M. Baker et al., "Common variation in the miR-659 binding-site of GRN is a major risk factor for TDP43-positive frontotemporal dementia," Human Molecular Genetics, vol. 17, no. 23, pp. 3631-3642, 2008.

[15] W. X. Wang, B. R. Wilfred, S. K. Madathil et al., "miR-107 regulates granulin/progranulin with implications for traumatic brain injury and neurodegenerative disease," American Journal of Pathology, vol. 177, no. 1, pp. 334-345, 2010.

[16] A. H. Williams, G. Valdez, V. Moresi et al., "MicroRNA206 delays ALS progression and promotes regeneration of neuromuscular synapses in mice," Science, vol. 326, no. 5959, pp. 1549-1554, 2009.

[17] R. Saba and G. M. Schratt, "MicroRNAs in neuronal development, function and dysfunction," Brain Research, vol. 1338, no. C, pp. 3-13, 2010.

[18] R. Fiore, G. Siegel, and G. Schratt, "MicroRNA function in neuronal development, plasticity and disease," Biochimica et Biophysica Acta, vol. 1779, no. 8, pp. 471-478, 2008.

[19] J. Krol, I. Loedige, and W. Filipowicz, "The widespread regulation of microRNA biogenesis, function and decay," Nature Reviews Genetics, vol. 11, no. 9, pp. 597-610, 2010.

[20] K. Breving and A. Esquela-Kerscher, "The complexities of microRNA regulation: mirandering around the rules," International Journal of Biochemistry and Cell Biology, vol. 42, no. 8, pp. 1316-1329, 2010.

[21] S. S. Hébert and B. De Strooper, "Alterations of the microRNA network cause neurodegenerative disease," Trends in Neurosciences, vol. 32, no. 4, pp. 199-206, 2009.

[22] M. P. Perron and P. Provost, "Protein interactions and complexes in human microRNA biogenesis and function," Frontiers in Bioscience, vol. 13, no. 7, pp. 2537-2547, 2008.

[23] V. Ambros, "The functions of animal microRNAs," Nature, vol. 431, no. 7006, pp. 350-355, 2004.

[24] S. Djuranovic, A. Nahvi, and R. Green, "A parsimonious model for gene regulation by miRNAs," Science, vol. 331, no. 6017, pp. 550-553, 2011.

[25] O. Hobert, "Gene regulation by transcription factors and MicroRNAs," Science, vol. 319, no. 5871, pp. 1785-1786, 2008.

[26] S. S. Hébert, A. S. Papadopoulou, P. Smith et al., "Genetic ablation of dicer in adult forebrain neurons results in abnormal tau hyperphosphorylation and neurodegeneration," Human Molecular Genetics, vol. 19, no. 20, pp. 3959-3969, 2010.

[27] A. Schaefer, D. O'Carroll, L. T. Chan et al., "Cerebellar neurodegeneration in the absence of microRNAs," Journal of Experimental Medicine, vol. 204, no. 7, pp. 1553-1558, 2007.

[28] T. L. Cuellar, T. H. Davis, P. T. Nelson et al., "Dicer loss in striatal neurons produces behavioral and neuroanatomical phenotypes in the absence of neurodegeneration," Proceedings of the National Academy of Sciences of the United States of America, vol. 105, no. 14, pp. 5614-5619, 2008.

[29] W. J. Lukiw, "Micro-RNA speciation in fetal, adult and Alzheimer's disease hippocampus," NeuroReport, vol. 18, no. 3, pp. 297-300, 2007.

[30] J. Nunez-Iglesias, C. C. Liu, T. E. Morgan, C. E. Finch, and X. J. Zhou, "Joint genome-wide profiling of miRNA and mRNA expression in Alzheimer's disease cortex reveals altered miRNA regulation," PloS One, vol. 5, no. 2, Article ID e8898, 2010.

[31] M. Shioya, S. Obayashi, H. Tabunoki et al., "Aberrant microRNA expression in the brains of neurodegenerative diseases: MiR-29a decreased in Alzheimer disease brains targets neurone navigator 3," Neuropathology and Applied Neurobiology, vol. 36, no. 4, pp. 320-330, 2010.

[32] J. P. Cogswell, J. Ward, I. A. Taylor et al., "Identification of miRNA changes in Alzheimer's disease brain and CSF yields putative biomarkers and insights into disease pathways," Journal of Alzheimer's Disease, vol. 14, no. 1, pp. 27-41, 2008.

[33] H. M. Schipper, O. C. Maes, H. M. Chertkow, and E. Wang, "MicroRNA expression in Alzheimer blood mononuclear cells," Gene Regulation and Systems Biology, vol. 1, pp. 12631274, 2007.

[34] B. John, A. J. Enright, A. Aravin, T. Tuschl, C. Sander, and D. S. Marks, "Human microRNA targets," PLoS Biology, vol. 2, no. 11, article e363, 2004.

[35] P. T. Nelson, W. X. Wang, and B. W. Rajeev, "MicroRNAs (miRNAs) in neurodegenerative diseases," Brain Pathology, vol. 18, no. 1, pp. 130-138, 2008.

[36] N. Patel, D. Hoang, N. Miller et al., "MicroRNAs can regulate human APP levels," Molecular Neurodegeneration, vol. 3, no. 1, article 10, 2008.

[37] H. Fukumoto, B. S. Cheung, B. T. Hyman, and M. C. Irizarry, " $\beta$-secretase protein and activity are increased in the neocortex in Alzheimer disease," Archives of Neurology, vol. 59, no. 9, pp. 1381-1389, 2002.

[38] R. M. D. Holsinger, C. A. McLean, K. Beyreuther, C. L. Masters, and G. Evin, "Increased expression of the amyloid precursor $\beta$-secretase in Alzheimer's disease," Annals of Neurology, vol. 51, no. 6, pp. 783-786, 2002.

[39] P. Achard, A. Herr, D. C. Baulcombe, and N. P. Harberd, "Modulation of floral development by a gibberellin-regulated microRNA," Development, vol. 131, no. 14, pp. 3357-3365, 2004.

[40] S. S. Hébert, K. Horré, L. Nicolaï et al., "MicroRNA regulation of Alzheimer's Amyloid precursor protein expression," Neurobiology of Disease, vol. 33, no. 3, pp. 422-428, 2009.

[41] T. H. Davis, T. L. Cuellar, S. M. Koch et al., "Conditional loss of dicer disrupts cellular and tissue morphogenesis in the cortex and hippocampus," Journal of Neuroscience, vol. 28, no. 17, pp. 4322-4330, 2008.

[42] Y. Kawase-Koga, R. Low, G. Otaegi et al., "RNAase-III enzyme Dicer maintains signaling pathways for differentiation and survival in mouse cortical neural stem cells," Journal of Cell Science, vol. 123, no. 4, pp. 586-594, 2010.

[43] J. Bilen, N. Liu, B. G. Burnett, R. N. Pittman, and N. M. Bonini, "MicroRNA pathways modulate polyglutamineinduced neurodegeneration," Molecular Cell, vol. 24, no. 1, pp. 157-163, 2006.

[44] D. Shin, J. Y. Shin, M. T. McManus, L. J. Ptáček, and Y. H. $\mathrm{Fu}$, "Dicer ablation in oligodendrocytes provokes neuronal impairment in mice," Annals of Neurology, vol. 66, no. 6, pp. 843-857, 2009. 
[45] W. Konopka, A. Kiryk, M. Novak et al., "MicroRNA loss enhances learning and memory in mice," Journal of Neuroscience, vol. 30, no. 44, pp. 14835-14842, 2010.

[46] M. Fabbri, R. Garzon, A. Cimmino et al., "MicroRNA29 family reverts aberrant methylation in lung cancer by targeting DNA methyltransferases 3A and 3B," Proceedings of the National Academy of Sciences of the United States of America, vol. 104, no. 40, pp. 15805-15810, 2007.

[47] A. J. Kole, V. Swahari, S. M. Hammond, and M. Deshmukh, "miR-29b is activated during neuronal maturation and targets BH3-only genes to restrict apoptosis," Genes and Development, vol. 25, no. 2, pp. 125-130, 2011.

[48] X. Wang, P. Liu, H. Zhu et al., "miR-34a, a microRNA upregulated in a double transgenic mouse model of Alzheimer's disease, inhibits bcl 2 translation," Brain Research Bulletin, vol. 80, no. 4-5, pp. 268-273, 2009.

[49] W.-X. Wang, Q. Huang, Y. Hu, A. J. Stromberg, and P. T. Nelson, "Patterns of microRNA expression in normal and early Alzheimer's disease human temporal cortex: white matter versus gray matter," Acta Neuropathologica, vol. 121, no. 2, pp. 193-205, 2011.

[50] Y. Zong, H. Wang, W. Dong et al., "miR-29c regulates BACE1 protein expression," Brain Research, vol. 1395, pp. 108-115, 2011.

[51] N. Schonrock, Y. D. Ke, D. Humphreys et al., "Neuronal microRNA deregulation in response to Alzheimer's disease amyloid-beta," PloS One, vol. 5, no. 6, Article ID e11070, 2010.

[52] J. Yao, T. Hennessey, A. Flynt, E. Lai, M. Flint Beal, and M. T. Lin, "MicroRNA-related cofilin abnormality in Alzheimer's disease," PLoS One, vol. 5, no. 12, Article ID e15546, 2010.

[53] J. Shen and R. J. Kelleher, "The presenilin hypothesis of Alzheimer's disease: evidence for a loss-of-function pathogenic mechanism," Proceedings of the National Academy of Sciences of the United States of America, vol. 104, no. 2, pp. 403-409, 2007.

[54] A. M. Krichevsky, K. S. King, C. P. Donahue, K. Khrapko, and K. S. Kosik, "A microRNA array reveals extensive regulation of microRNAs during brain development," RNA, vol. 9, no. 10, pp. 1274-1281, 2003.

[55] L. Jing, Y. Jya, J. Lu et al., "MicroRNA-9 promotes differentiation of mouse bone mesenchymal stem cells into neurons by Notch signaling," NeuroReport, vol. 22, no. 5, pp. 206-211, 2011.

[56] B. Bonev, A. Pisco, and N. Papalopulu, "MicroRNA-9 reveals regional diversity of neural progenitors along the anteriorposterior axis," Developmental Cell, vol. 20, no. 1, pp. 19-32, 2011.

[57] Y. Y. Li, J. G. Cui, J. M. Hill, S. Bhattacharjee, Y. Zhao, and W. J. Lukiw, "Increased expression of miRNA-146a in Alzheimer's disease transgenic mouse models," Neuroscience Letters, vol. 487, no. 1, pp. 94-98, 2011.

[58] W. J. Lukiw, Y. Zhao, and G. C. Jian, "An NF- $\kappa$ B-sensitive micro RNA-146a-mediated inflammatory circuit in alzheimer disease and in stressed human brain cells," Journal of Biological Chemistry, vol. 283, no. 46, pp. 31315-31322, 2008.

[59] L. Li, X.-P. Chen, and Y.-J. Li, "MicroRNA-146a and human disease," Scandinavian Journal of Immunology, vol. 71, no. 4, pp. 227-231, 2010.

[60] Y. J. Lee, S. B. Han, S. Y. Nam, K. W. Oh, and J. T. Hong, "Inflammation and Alzheimer's disease," Archives of Pharmacal Research, vol. 33, no. 10, pp. 1539-1556, 2010.

[61] S. W. Chi, J. B. Zang, A. Mele, and R. B. Darnell, "Argonaute HITS-CLIP decodes microRNA-mRNA interaction maps," Nature, vol. 460, no. 7254, pp. 479-486, 2009.
[62] J. Vinther, M. M. Hedegaard, P. P. Gardner, J. S. Andersen, and P. Arctander, "Identification of miRNA targets with stable isotope labeling by amino acids in cell culture," Nucleic Acids Research, vol. 34, no. 16, article e107, 2006.

[63] L. P. Lim, N. C. Lau, P. Garrett-Engele et al., "Microarray analysis shows that some microRNAs downregulate large numbers of-target mRNAs," Nature, vol. 433, no. 7027, pp. 769-773, 2005.

[64] N. Patel, D. Hoang, N. Miller et al., "MicroRNAs can regulate human APP levels," Molecular Neurodegeneration, vol. 3, no. 1, article 10, 2008.

[65] J. M. Long and D. K. Lahiri, "MicroRNA-101 downregulates Alzheimer's amyloid- $\beta$ precursor protein levels in human cell cultures and is differentially expressed," Biochemical and Biophysical Research Communications, vol. 404, no. 4, pp. 889895, 2011.

[66] X. Fan, Y. Liu, J. Jiang et al., "MiR-20a promotes proliferation and invasion by targeting APP in human ovarian cancer cells," Acta Biochimica et Biophysica Sinica, vol. 42, no. 5, pp. 318324, 2010.

[67] E. Vilardo, C. Barbato, M. Ciotti, C. Cogoni, and F. Ruberti, "MicroRNA-101 regulates amyloid precursor protein expression in hippocampal neurons," Journal of Biological Chemistry, vol. 285, no. 24, pp. 18344-18351, 2010.

[68] W. Liu, C. Liu, J. Zhu et al., "MicroRNA-16 targets amyloid precursor protein to potentially modulate Alzheimer'sassociated pathogenesis in SAMP8 mice," Neurobiology of Aging. In press.

[69] T. O'Connor, K. R. Sadleir, E. Maus et al., "Phosphorylation of the translation initiation factor eIF $2 \alpha$ increases BACE1 levels and promotes amyloidogenesis," Neuron, vol. 60, no. 6, pp. 988-1009, 2008.

[70] V. Boissonneault, I. Plante, S. Rivest, and P. Provost, "MicroRNA-298 and microRNA-328 regulate expression of mouse $\beta$-amyloid precursor protein-converting enzyme 1," Journal of Biological Chemistry, vol. 284, no. 4, pp. 1971-1981, 2009.

[71] M. A. Faghihi, F. Modarresi, A. M. Khalil et al., "Expression of a noncoding RNA is elevated in Alzheimer's disease and drives rapid feed-forward regulation of $\beta$-secretase," Nature Medicine, vol. 14, no. 7, pp. 723-730, 2008.

[72] J. Marksteiner, H. Hinterhuber, and C. Humpel, "Cerebrospinal fluid biomarkers for diagnosis of Alzheimer's disease: beta-amyloid(1-42), tau, phospho-tau-181 and total protein," Drugs of Today, vol. 43, no. 6, pp. 423-431, 2007.

[73] C. Reitz, C. Brayne, and R. Mayeux, "Epidemiology of Alzheimer disease," Nature Reviews Neurology, vol. 7, no. 3, pp. 137-152, 2011.

[74] M. Ciesla, K. Skrzypek, M. Kozakowska, A. Loboda, A. Jozkowicz, and J. Dulak, "MicroRNAs as biomarkers of disease onset," Analytical and Bioanalytical Chemistry. In press.

[75] H. Mizuno, A. Nakamura, Y. Aoki et al., "Identification of muscle-specific MicroRNAs in serum of muscular dystrophy animal models: promising novel blood-based markers for muscular dystrophy," PLoS One, vol. 6, no. 3, Article ID e18388, 2011.

[76] K. Wang, S. Zhang, B. Marzolf et al., "Circulating microRNAs, potential biomarkers for drug-induced liver injury," Proceedings of the National Academy of Sciences of the United States of America, vol. 106, no. 11, pp. 4402-4407, 2009. 


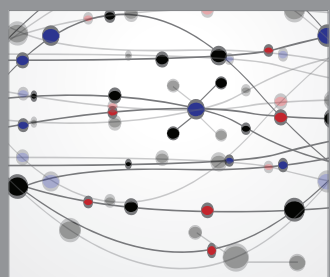

The Scientific World Journal
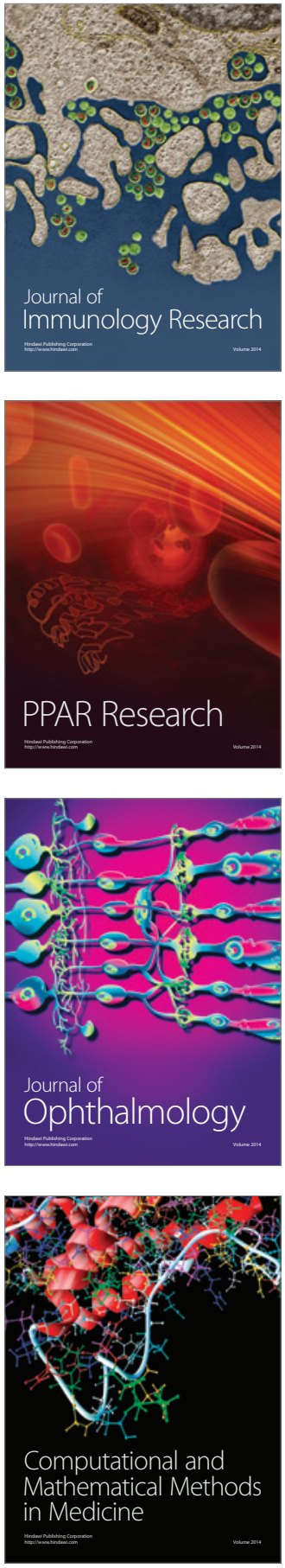

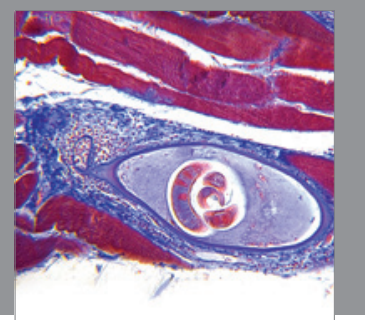

Gastroenterology

Research and Practice
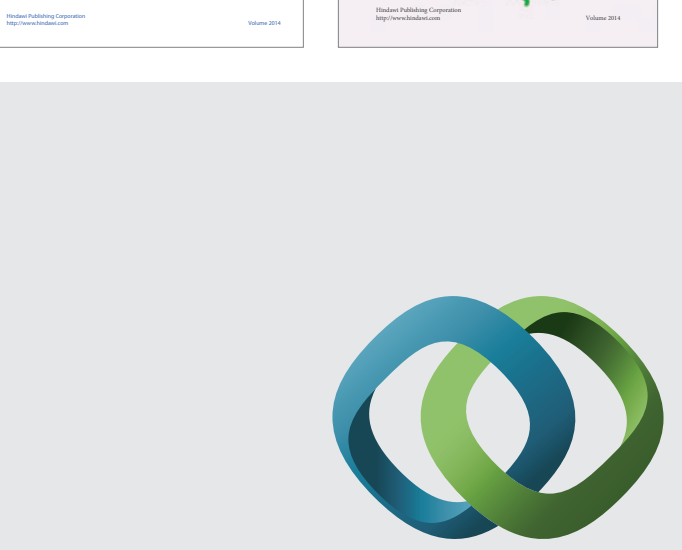

\section{Hindawi}

Submit your manuscripts at

http://www.hindawi.com
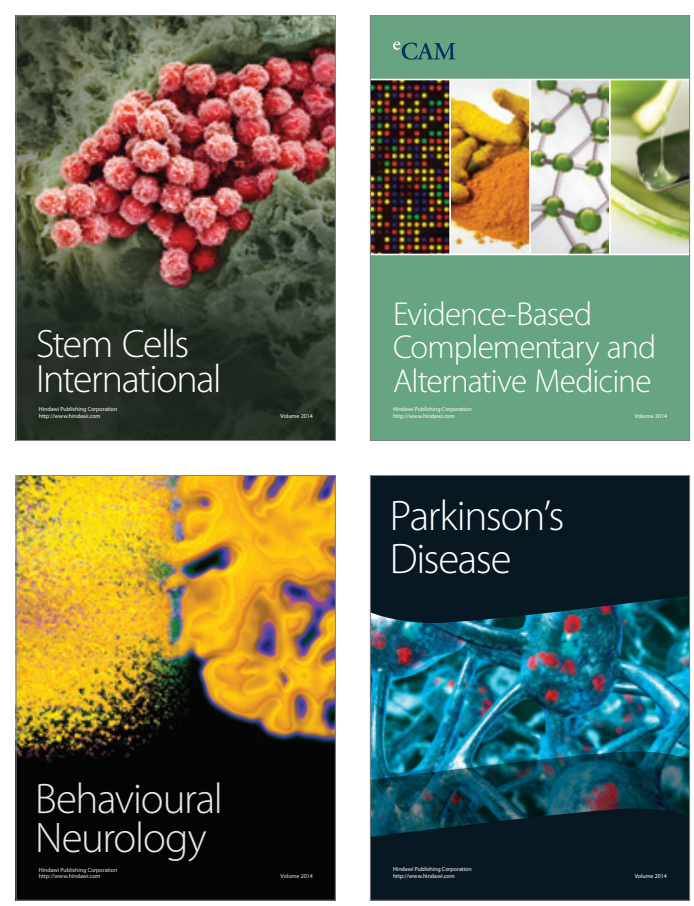

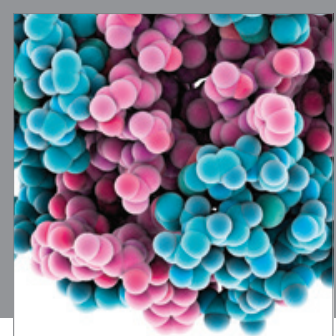

Journal of
Diabetes Research

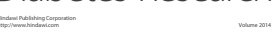

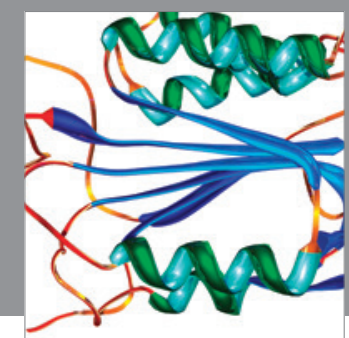

Disease Markers
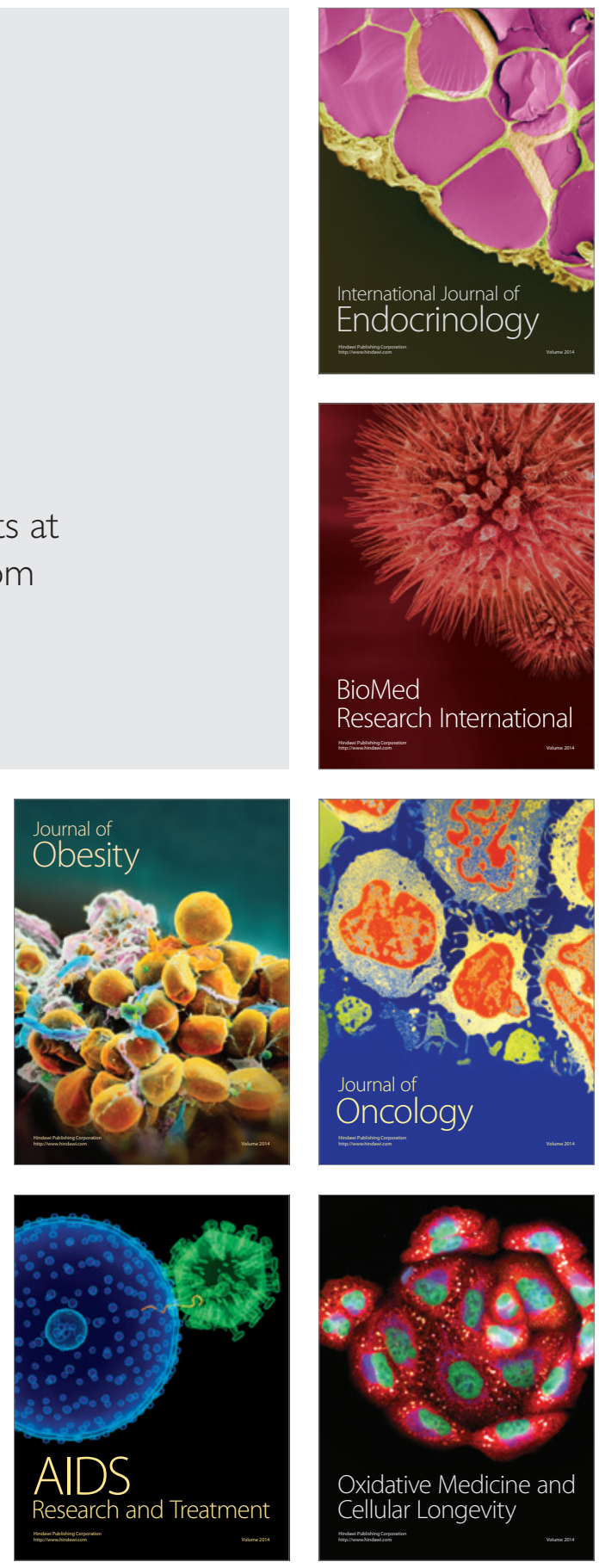